Research Article

\title{
Strong Duality and Optimality Conditions for Generalized Equilibrium Problems
}

\author{
D. H. Fang ${ }^{1,2}$ and J. F. Bao ${ }^{3}$ \\ ${ }^{1}$ College of Mathematics and Statistics, Jishou University, Jishou 416000, China \\ ${ }^{2}$ Department of Applied Mathematics, National Sun Yat-sen University, Kaohsiung 80424, Taiwan \\ ${ }^{3}$ School of Mathematics, Physics and Information Science, Zhejiang Ocean University, Zhoushan 316004, China
}

Correspondence should be addressed to D. H. Fang; dh_fang@163.com

Received 29 August 2013; Accepted 4 September 2013

Academic Editor: Jen-Chih Yao

Copyright (C) 2013 D. H. Fang and J. F. Bao. This is an open access article distributed under the Creative Commons Attribution License, which permits unrestricted use, distribution, and reproduction in any medium, provided the original work is properly cited.

We consider a generalized equilibrium problem involving DC functions. By using the properties of the epigraph of the conjugate functions, some sufficient and/or necessary conditions for the weak and strong duality results and optimality conditions for generalized equilibrium problems are provided.

\section{Introduction}

Consider the following generalized equilibrium problem:

$$
\begin{gathered}
\text { Find } x \in C \text { such that } \\
f(x, y)+\psi(y) \geq \psi(x) \quad \text { for each } y \in C,
\end{gathered}
$$

where $X$ is a locally convex Hausdorff topological space, $C$ is a nonempty convex subset of $X, f: X \times X \rightarrow \overline{\mathbb{R}}:=\mathbb{R} \cup\{+\infty\}$, and $\psi: X \rightarrow \overline{\mathbb{R}}$ are proper functions satisfying the following.

(a) $f(x, x)=0$ for all $x \in C$.

(b) $f_{x}(\cdot):=f(x, \cdot)$ is proper convex for all $x \in C$.

(c) $\psi:=g-h$, where $g, h: X \rightarrow \overline{\mathbb{R}}$ are two proper convex functions.

Here and throughout the whole paper, following [1, page 39], we adapt the convention that $(+\infty)+(-\infty)=(+\infty)-(+\infty)=$ $+\infty$.

As mentioned in [2], equilibrium problems theory provides us with a unified, natural, innovative, and general framework to study a wide class of problems arising in finance, economics, network analysis, transportation, elasticity, and optimization. This theory has witnessed an explosive growth in theoretical advances and applications across all disciplines of pure and applied sciences. Equilibrium problems have been studied extensively, and many problems such as optimization problems, Nash equilibria problems, complementarity problems, fixed point problems, variational inequality problems, and convex vector optimization problems can be recast into the form (GEP); see, for example, [3$10]$ and the references therein.

Duality for equilibrium problems was first studied in [11]. The schemes proposed in that paper are extensions of a classical duality theory for variational inequalities. In spirit of convex optimization, duality results and optimality conditions have been obtained for equilibrium problems by Martínez-Legaz and Sosa [12] when $\psi=0$ and by Jacinto and Scheimberg [13] when $\psi$ is convex, which extended the classical convex duality results. Recently, the authors in [5] considered the generalized equilibrium problems in the case where $\psi$ is a DC function. Under the assumptions that $C$ is closed and functions $f(\cdot, x), g, h$ are lower semicontinuous (lsc in brief), they gave some weak and strong duality results and optimality conditions for (GEP) via a closedness qualification condition.

Inspired by the works mentioned above, we continue to study the generalized equilibrium problems. Our main aim in the present paper is to give some new regularity conditions which characterize the weak duality, the strong duality, and optimality conditions for (GEP). In general, we do not impose 
any topological assumption on $C$ or on $f_{x}(\cdot), g$, and $h$; that is, $C$ is not necessarily closed, and $f_{x}(\cdot), g$, and $h$ are not necessarily lsc. Most of results obtained in the present paper are proper extensions of the early results (e.g., $[5,14-16])$. In particular, even in the special case when the closedness of $C$ and lower semicontinuity of $f_{x}(\cdot), g$, and $h$ are satisfied, our results provide improved versions of [5, Theorems 4.2, 4.3 and 4.4].

The paper is organized as follows. Section 2 contains some necessary notations and preliminary results. In Section 3, we develop general duality and optimality results for a DC optimization problem. The weak and strong duality results and optimality conditions for generalized equilibrium problems are given in Section 4.

\section{Notations and Preliminaries}

The notation used in the present paper is standard (cf. [1]). In particular, we assume throughout the whole paper that $X$ is a real locally convex Hausdorff topological vector space, and $X^{*}$ denotes the dual space, endowed with the weak ${ }^{*}$-topology $w^{*}\left(X^{*}, X\right)$. By $\left\langle x^{*}, x\right\rangle$ we will denote the value of the functional $x^{*} \in X^{*}$ at $x \in X$; that is, $\left\langle x^{*}, x\right\rangle=x^{*}(x)$. Let $Z$ be a set in $X$. The closure of $Z$ is denoted by $\operatorname{cl} Z$. If $W \subseteq X^{*}$, then $\mathrm{cl} W$ denotes the weak ${ }^{*}$-closure of $W$. For the whole paper, we endow $X^{*} \times \mathbb{R}$ with the product topology of $w^{*}\left(X^{*}, X\right)$ and the usual Euclidean topology.

The indicator function $\delta_{Z}$ of a nonempty set $Z$ is defined by $\delta_{Z}(x)=0$ if $x \in Z$ and $\delta_{Z}(x)=+\infty$ if $x \notin Z$. Let $f: X \rightarrow$ $\overline{\mathbb{R}}$ be a proper function. The conjugate function and the epigraph of $f$ are denoted by $f^{*}$ and epi $f$, respectively; they are defined by

$$
\begin{aligned}
f^{*}\left(x^{*}\right):= & \sup _{x \in \operatorname{dom} f}\left\{\left\langle x^{*}, x\right\rangle-f(x)\right\} \quad \text { for each } x^{*} \in X^{*}, \\
& \text { epi } f:=\{(x, r) \in X \times \mathbb{R}: f(x) \leq r\},
\end{aligned}
$$

where the effective domain dom $f:=\{x \in X: f(x)<+\infty\}$. It is well known and easy to verify that epi $f^{*}$ is weak ${ }^{*}$-closed. The lsc hull of $f$, denoted by $\mathrm{cl} f$, is defined by

$$
\operatorname{epi}(\operatorname{cl} f)=\operatorname{cl}(\operatorname{epi} f) \text {. }
$$

Then, by [1, Theorem 2.3.1], $f^{*}=(\operatorname{cl} f)^{*}$ and by [1, Theorem 2.3.4], if $\mathrm{cl} f$ is proper and convex, then $f^{* *}=\mathrm{cl} f$. Let $x \in X$. The subdifferential of $f$ at $x$ is defined by

$$
\begin{gathered}
\partial f(x):=\left\{x^{*} \in X^{*}: f(x)+\left\langle x^{*}, y-x\right\rangle \leq f(y)\right. \\
\text { for each } y \in X\}
\end{gathered}
$$

if $x \in \operatorname{dom} f$, and $\partial f(x):=\emptyset$ otherwise. Clearly, the following equivalence holds:

$$
x_{0} \text { is a minimizer of } f \text { iff } 0 \in \partial f\left(x_{0}\right) .
$$

By definition, the Young-Fenchel inequality below holds:

$$
\begin{aligned}
& f(x)+f^{*}\left(x^{*}\right) \\
& \geq\left\langle x, x^{*}\right\rangle \text { for each pair }\left(x, x^{*}\right) \in X \times X^{*} .
\end{aligned}
$$

Moreover, by [1, Theorem 2.4.2(iii)],

$$
f(x)+f^{*}\left(x^{*}\right)=\left\langle x^{*}, x\right\rangle \text { iff } x^{*} \in \partial f(x)
$$

(the equality in (7) is usually referred to as Young's equality). If $g, h$ are proper, then

$$
\begin{gathered}
\text { epi } g^{*}+\text { epi } h^{*} \subseteq \text { epi }(g+h)^{*}, \\
g \leq h \Longrightarrow g^{*} \geq h^{*} \Longleftrightarrow \operatorname{epi} g^{*} \subseteq \text { epi } h^{*}, \\
\partial g(a)+\partial h(a) \subseteq \partial(g+h)(a) \\
\text { for each } a \in \operatorname{dom} g \cap \operatorname{dom} h .
\end{gathered}
$$

Furthermore, for each $p \in X^{*}$ and $a \in \mathbb{R}$,

$$
\text { epi }(h+p+a)^{*}=\operatorname{epi} h^{*}+(p,-a) \text {. }
$$

Moreover, if $g$ is convex and $\operatorname{lsc}$ on $\operatorname{dom} h$, then, by [17, Lemma 2.3],

$$
\operatorname{epi}(h-g)^{*}=\bigcap_{x^{*} \in \operatorname{dom} g^{*}}\left(\operatorname{epi} h^{*}-\left(x^{*}, g^{*}\left(x^{*}\right)\right)\right) \text {. }
$$

The following lemma is known in $[1,6]$ (cf. [6, Lemma 2.1] for (11) and (12) and [1, Theorem 2.8.7] for (13)).

Lemma 1. Let $g, h: X \rightarrow \overline{\mathbb{R}}$ be proper convex functions satisfying dom $g \cap \operatorname{dom} h \neq \emptyset$.

(i) If $g, h$ are $l s c$, then

$$
\text { epi }(g+h)^{*}=\operatorname{cl}\left(\text { epi } g^{*}+\text { epi } h^{*}\right) \text {. }
$$

(ii) If either $g$ or $h$ is continuous at some point of dom $g \cap$ dom $h$, then

$$
\begin{aligned}
& \text { epi }(g+h)^{*}=\text { epi } g^{*}+\text { epi } h^{*}, \\
& \partial(g+h)(x) \\
& \quad=\partial g(x)+\partial h(x) \quad \text { for each } x \in \operatorname{dom} g \cap \operatorname{dom} h .
\end{aligned}
$$

\section{Duality and Optimality Conditions for DC Optimization Problem}

Let $p \in X^{*}$. Consider the following DC optimization problem:

$$
\left(P_{p}\right) \quad \inf _{y \in C}\{F(y)+G(y)-H(y)-\langle p, y\rangle\},
$$

where $C$ is a convex subset of $X$ (not necessarily closed) and $F, G, H: X \rightarrow \overline{\mathbb{R}}$ are proper convex functions (not necessaily lsc). Following [5], we define the dual problem of $\left(P_{p}\right)$ by

$$
\left(D_{p}\right) \inf _{x^{*} \in \operatorname{dom} H^{*}} \sup _{u^{*} \in \operatorname{dom} F^{*}, v^{*} \in \operatorname{dom} G^{*}} L_{p}\left(x^{*}, u^{*}, v^{*}\right),
$$

where $L_{p}: \operatorname{dom} H^{*} \times \operatorname{dom} F^{*} \times \operatorname{dom} G^{*} \rightarrow \overline{\mathbb{R}}$ is defined by

$$
\begin{aligned}
L_{p}\left(x^{*}, u^{*}, v^{*}\right):= & H^{*}\left(x^{*}\right)-F^{*}\left(p+u^{*}\right) \\
& -G^{*}\left(v^{*}\right)-\delta_{C}^{*}\left(x^{*}-u^{*}-v^{*}\right) .
\end{aligned}
$$


Let $v\left(P_{p}\right)$ and $v\left(D_{p}\right)$ denote the optimal values of problems $\left(P_{p}\right)$ and $\left(D_{p}\right)$, respectively. Especially, in the case when $p=$ 0 , we write $(P),(D)$ and $L(\cdot, \cdot, \cdot)$ for $\left(P_{0}\right),\left(D_{0}\right)$, and $L_{0}(\cdot, \cdot, \cdot)$, respectively. One of the main aims in this section is devoted to the study of the weak duality and the strong duality between $(P)$ and $(D)$, which are defined as follows.

\section{Definition 2. We say that}

(a) the weak duality holds (between $(P)$ and $(D)$ ) if $v(D) \leq v(P)$

(b) the strong duality holds (between $(P)$ and $(D)$ ) if $v(P)=v(D)$ and for each $x^{*} \in \operatorname{dom} F^{*}$, there exists $\left(u^{*}, v^{*}\right) \in \operatorname{dom} F^{*} \times \operatorname{dom} G^{*}$ such that $L\left(x^{*}, u^{*}, v^{*}\right) \geq$ $v(D)$;

(c) the stable weak duality (resp., the stable strong duality) holds if the weak duality (resp., the strong duality) holds between $\left(P_{p}\right)$ and $\left(D_{p}\right)$ for each $p \in X^{*}$.

If $H$ is lsc, then by [5, Theorem 3.2(i)], the weak duality holds. However, the weak duality does not necessarily hold in general as will be shown in the following example.

Example 3. Let $X:=\mathbb{R}$ and $C:=[0,+\infty)$. Let $F, G, H: \mathbb{R} \rightarrow$ $\overline{\mathbb{R}}$ be defined by $F:=0, G:=\delta_{[0,+\infty)}$, and

$$
H(y):= \begin{cases}0 & y<0 \\ 1 & y=0 \\ +\infty & y>0\end{cases}
$$

Then, $F, G$, and $H$ are proper convex functions and $v(P)=-1$. Clearly, $F^{*}=\delta_{\{0\}}, G^{*}=\delta_{C}^{*}=\delta_{(-\infty, 0]}$, and $H^{*}=\delta_{[0,+\infty)}$. Hence, $v(D)=0$. This implies that $v(D)>v(P)$. Consequently, the weak duality does not hold.

To consider the weak duality, the strong duality, and optimality conditions for problem $(P)$, we introduced the following conditions. For simplicity, we denote

$$
\begin{aligned}
K:=\bigcap_{x^{*} \in \operatorname{dom} H^{*}}\left(\text { epi } F^{*}+\text { epi } G^{*}\right. & \\
& \left.+\operatorname{epi} \delta_{C}^{*}-\left(x^{*}, H^{*}\left(x^{*}\right)\right)\right),
\end{aligned}
$$

where we adapt the convention $\cap_{t \in \emptyset} S_{t}=X$.

Definition 4 . The family $\left\{F, G, H, \delta_{C}\right\}$ is said to satisfy

(i) the weak closure condition at $0\left((\mathrm{WCC})_{0}\right)$ if

$K \cap(\{0\} \times \mathbb{R}) \subseteq \operatorname{epi}\left(F+G-H+\delta_{C}\right)^{*} \cap(\{0\} \times \mathbb{R}) ;$

(ii) the closure condition at $0\left((\mathrm{CC})_{0}\right)$ if

$$
K \cap(\{0\} \times \mathbb{R})=\operatorname{epi}\left(F+G-H+\delta_{C}\right)^{*} \cap(\{0\} \times \mathbb{R}) ;
$$

(iii) the weak closure condition ((WCC)) if

$$
K \subseteq \operatorname{epi}\left(F+G-H+\delta_{C}\right)^{*} ;
$$

(iv) the closure condition ((CC)) if

$$
K=\operatorname{epi}\left(F+G-H+\delta_{C}\right)^{*}
$$

(v) the Moreau-Rockafellar formula (MRF) at $x \in$ $\operatorname{dom}(F+G-H) \cap C$ if

$$
\begin{aligned}
& \partial\left(F+G-H+\delta_{C}\right)(x) \\
& \quad \subseteq \bigcap_{x^{*} \in \partial H(x)}\left(\partial F(x)+\partial G(x)+N_{C}(x)-x^{*}\right) ;
\end{aligned}
$$

(vi) (MRF) if it satisfies (MRF) at each point in $\operatorname{dom}(F+$ $G-H) \cap C$.

Remark 5. If $H$ is lsc, then by (10), we have that

$$
K=\operatorname{epi}(F-H)^{*}+\text { epi } G^{*}+\operatorname{epi} \delta_{C}^{*}
$$

and, by (8), (21) holds; that is, the (WCC) holds.

The following proposition describes the relationship between the (CC) (resp., the (WCC)) and the (CC) $)_{0}$ (resp., the $\left.(\mathrm{WCC})_{0}\right)$.

Proposition 6. The family $\left\{F, G, H, \delta_{C}\right\}$ satisfies the (CC) (resp., the (WCC)) if and only if for each $p \in X^{*},\{F-p, G, H$, $\left.\delta_{C}\right\}$ satisfies the $(C C)_{0}\left(\right.$ resp., the $\left.(W C C)_{0}\right)$.

Proof. Let $p \in X^{*}$, and let $K(p)$ be the set defined by

$$
\begin{aligned}
K(p):=\bigcap_{x^{*} \in \operatorname{dom} H^{*}}\left(\operatorname{epi}(F-p)^{*}+\operatorname{epi} G^{*}\right. \\
\\
\left.+\operatorname{epi} \delta_{C}^{*}-\left(x^{*}, H^{*}\left(x^{*}\right)\right)\right) .
\end{aligned}
$$

Then, by (9), the following equality is clear:

$$
K(p)=K+(-p, 0) .
$$

Hence, we have that

$$
K(p) \cap(\{0\} \times \mathbb{R})=K \cap(\{p\} \times \mathbb{R})+(-p, 0) .
$$

Moreover, using (9), we conclude that

$$
\begin{aligned}
& \operatorname{epi}\left(F-p+G-H+\delta_{C}\right)^{*} \cap(\{0\} \times \mathbb{R}) \\
& \quad=\operatorname{epi}\left(F+G-H+\delta_{C}\right)^{*} \cap(\{p\} \times \mathbb{R})+(-p, 0) .
\end{aligned}
$$

Thus, the conclusion holds by definitions and the proof is complete.

Under the assumption that

$$
F, G, H \text { are lsc and } C \text { is closed, }
$$

the authors in [5] introduced the following closure condition

$$
\text { epi } F^{*}+\text { epi } G^{*}+\text { epi } \delta_{C}^{*} \quad \text { is } \text { weak }^{*} \text {-closed }
$$

to consider the strong duality and optimality conditions for DC optimization problem (14). The following proposition describes the relationships among the (CC), the (MRF), and (30). 
Proposition 7. The following implication holds:

$$
(C C) \Longrightarrow(M R F) \text {. }
$$

Furthermore, if (29) holds, then the following implications hold:

$$
(30) \Longrightarrow(C C) \Longrightarrow(M R F) \text {. }
$$

Proof. Suppose that the (CC) holds. Let $x_{0} \in A$, and let $p \in$ $\partial\left(F+G-H+\delta_{C}\right)\left(x_{0}\right)$. Then, by $(7)$,

$$
\begin{gathered}
\left(p,\left\langle p, x_{0}\right\rangle-\left(F+G-H+\delta_{C}\right)\left(x_{0}\right)\right) \\
\in \operatorname{epi}\left(F+G-H+\delta_{C}\right)^{*}=K,
\end{gathered}
$$

thanks to the (CC). Hence, for each $x^{*} \in \partial H\left(x_{0}\right)$,

$$
\begin{aligned}
& \left(p,\left\langle p, x_{0}\right\rangle-\left(F+G-H+\delta_{C}\right)\left(x_{0}\right)\right) \\
& \quad \in \operatorname{epi} F^{*}+\operatorname{epi} G^{*}+\operatorname{epi} \delta_{C}^{*}-\left(x^{*}, H^{*}\left(x^{*}\right)\right) .
\end{aligned}
$$

Let $x^{*} \in \partial H\left(x_{0}\right)$. Then, there exist $\left(u^{*}, r_{1}\right) \in \operatorname{epi} F^{*},\left(v^{*}, r_{2}\right) \in$ epi $G^{*}$, and $\left(w^{*}, r_{3}\right) \in$ epi $\delta_{C}^{*}$ such that

$$
\begin{gathered}
u^{*}+v^{*}+w^{*}-x^{*}=p, \\
r_{1}+r_{2}+r_{3}-H^{*}\left(x^{*}\right)=\left\langle p, x_{0}\right\rangle-\left(F+G-H+\delta_{C}\right)\left(x_{0}\right) .
\end{gathered}
$$

Below we show that $u^{*} \in \partial F\left(x_{0}\right), v^{*} \in \partial G\left(x_{0}\right)$, and $w^{*} \in$ $N_{C}\left(x_{0}\right)$. To do this, note by the definition that

$$
F^{*}\left(u^{*}\right) \leq r_{1}, \quad G^{*}\left(v^{*}\right) \leq r_{2}, \quad \delta_{C}^{*}\left(w^{*}\right) \leq r_{3} .
$$

Moreover, since $x^{*} \in \partial H\left(x_{0}\right)$, it follows from (7) that

$$
H\left(x_{0}\right)+H^{*}\left(x^{*}\right)=\left\langle x^{*}, x_{0}\right\rangle .
$$

Hence, by (35)-(37) and the Young-Fenchel inequality (6), we have that

$$
\begin{aligned}
0 \leq & F^{*}\left(u^{*}\right)+F\left(x_{0}\right)-\left\langle u^{*}, x_{0}\right\rangle \\
\leq & F^{*}\left(u^{*}\right)+F\left(x_{0}\right)-\left\langle x^{*}+p-v^{*}-w^{*}, x_{0}\right\rangle \\
\leq & F^{*}\left(u^{*}\right)-r_{1}+\left(\left\langle v^{*}, x_{0}\right\rangle-G\left(x_{0}\right)-r_{2}\right) \\
& +\left(\left\langle w^{*}, x_{0}\right\rangle-\delta_{C}\left(x_{0}\right)-r_{3}\right) \\
& +\left(H\left(x_{0}\right)+H^{*}\left(x^{*}\right)-\left\langle x^{*}, x_{0}\right\rangle\right) \\
\leq & \left(F^{*}\left(u^{*}\right)-r_{1}\right)+\left(G^{*}\left(v^{*}\right)-r_{2}\right)+\left(\delta_{C}^{*}\left(w^{*}\right)-r_{3}\right) \\
\leq & 0 .
\end{aligned}
$$

Thus,

$$
F^{*}\left(u^{*}\right)+F\left(x_{0}\right)-\left\langle u^{*}, x_{0}\right\rangle=0 .
$$

This implies that $u^{*} \in \partial F\left(x_{0}\right)$ by (7). Using the same argument, we have that $v^{*} \in \partial G\left(x_{0}\right)$ and $w^{*} \in N_{C}\left(x_{0}\right)$. Hence, $p \in \partial F\left(x_{0}\right)+\partial G\left(x_{0}\right)+N_{C}\left(x_{0}\right)-x^{*}$, and

$$
p \in \bigcap_{x^{*} \in \partial H\left(x_{0}\right)}\left(\partial F\left(x_{0}\right)+\partial G\left(x_{0}\right)+N_{C}\left(x_{0}\right)-x^{*}\right)
$$

since $x^{*} \in \partial H\left(x_{0}\right)$ is arbitrary. Therefore, the (MRF) holds.
Furthermore, suppose that (29) holds. To show that (32), we only need to show the implication $(30) \Rightarrow$ (CC) holds. To do this, we assume that (30) holds. Since $H$ is lsc, it follows from (10) that

$$
\begin{aligned}
\operatorname{epi} & \left(F+G+\delta_{C}-H\right)^{*} \\
& =\bigcap_{x^{*} \in \operatorname{dom} H^{*}}\left(\operatorname{epi}\left(F+G+\delta_{C}\right)^{*}-\left(x^{*}, H^{*}\left(x^{*}\right)\right)\right) .
\end{aligned}
$$

Note that $F, G$ are lsc and $C$ is closed; by Lemma 1(i), one has that

$$
\operatorname{epi}\left(F+G+\delta_{C}\right)^{*}=\operatorname{cl}\left(\text { epi } F^{*}+\text { epi } G^{*}+\text { epi } \delta_{C}^{*}\right) \text {. }
$$

This together with (30) and (41) implies that the (CC) holds. The proof is complete.

To study the weak duality and the strong duality, we need the following lemma.

Lemma 8. Let $r \in \mathbb{R}$. Then, the following assertions hold:

(i) $(0, r) \in \operatorname{epi}\left(F+G-H+\delta_{C}\right)^{*}$ if and only if $v(P) \geq-r$.

(ii) $(0, r) \in K$ if and only if $v(D) \geq-r$ and for each $x^{*} \epsilon$ $\operatorname{dom} H^{*}$, there exist $u^{*} \in \operatorname{dom} F^{*}$ and $v^{*} \in \operatorname{dom} G^{*}$ such that

$$
L\left(x^{*}, u^{*}, v^{*}\right) \geq-r .
$$

Proof. (i) By the definition of the conjugate function, one has

$$
v(P)=-\left(F+G-H+\delta_{C}\right)^{*}(0) .
$$

Hence, the result is clear.

(ii) Let $(0, r) \in K$ and let $x^{*} \in \operatorname{dom} H^{*}$. Then

$$
(0, r) \in \text { epi } F^{*}+\text { epi } G^{*}+\text { epi } \delta_{C}^{*}-\left(x^{*}, H^{*}\left(x^{*}\right)\right) .
$$

Thus, there exist $\left(u^{*}, r_{1}\right) \in$ epi $F^{*},\left(v^{*}, r_{2}\right) \in$ epi $G^{*}$, and $\left(w^{*}, r_{3}\right) \in$ epi $\delta_{C}^{*}$ such that

$$
\begin{gathered}
u^{*}+v^{*}+w^{*}-x^{*}=0, \\
r_{1}+r_{2}+r_{3}-H^{*}\left(x^{*}\right)=r .
\end{gathered}
$$

Since

$$
F^{*}\left(u^{*}\right) \leq r_{1}, \quad G^{*}\left(v^{*}\right) \leq r_{2}, \quad \delta_{C}^{*}\left(w^{*}\right) \leq r_{3},
$$

it follows from (46) that

$$
\begin{aligned}
L\left(x^{*}, u^{*}, v^{*}\right)= & H^{*}\left(x^{*}\right)-F^{*}\left(u^{*}\right)-G^{*}\left(v^{*}\right) \\
& -\delta_{C}^{*}\left(x^{*}-u^{*}-v^{*}\right) \\
\geq & H^{*}\left(x^{*}\right)-r_{1}-r_{2}-r_{3} \\
= & -r .
\end{aligned}
$$

This together with the definition of $v(D)$ implies that $v(D) \geq$ $-r$ and $u^{*}, v^{*}$ satisfy (43). 
Conversely, suppose that $v(D) \geq-r$ and for each $x^{*} \in$ $\operatorname{dom} H^{*}$, there exist $u^{*} \in \operatorname{dom} F^{*}$ and $v^{*} \in \operatorname{dom} G^{*}$ satisfying (43). Let $x^{*} \in \operatorname{dom} H^{*}$. Then, there exist $u^{*} \in \operatorname{dom} F^{*}$ and $v^{*} \in \operatorname{dom} G^{*}$ such that (43) holds. Then

$$
\delta_{C}^{*}\left(x^{*}-u^{*}-v^{*}\right) \leq r+H^{*}\left(x^{*}\right)-F^{*}\left(u^{*}\right)-G^{*}\left(v^{*}\right) .
$$

This means that

$$
\left(x^{*}-u^{*}-v^{*}, r+H^{*}\left(x^{*}\right)-F^{*}\left(u^{*}\right)-G^{*}\left(v^{*}\right)\right) \in \operatorname{epi} \delta_{C}^{*} .
$$

Therefore,

$$
\begin{aligned}
(0, r)= & \left(u^{*}, F^{*}\left(u^{*}\right)\right)+\left(v^{*}, G^{*}\left(v^{*}\right)\right) \\
& -\left(x^{*}, H^{*}\left(x^{*}\right)\right) \\
& +\left(x^{*}-u^{*}-v^{*}, r+H^{*}\left(x^{*}\right)-F^{*}\left(u^{*}\right)-G^{*}\left(v^{*}\right)\right) \\
\epsilon & \text { epi } F^{*}+\text { epi } G+\operatorname{epi} \delta_{C}^{*}-\left(x^{*}, H^{*}\left(x^{*}\right)\right) .
\end{aligned}
$$

Noting that $x^{*} \in \operatorname{dom} H^{*}$ is arbitrary, we have that

$$
\begin{aligned}
(0, r) \in \bigcap_{x^{*} \in \operatorname{dom} H^{*}}\left(\text { epi } F^{*}+\text { epi } G\right. & \\
& \left.+\operatorname{epi} \delta_{C}^{*}-\left(x^{*}, H^{*}\left(x^{*}\right)\right)\right)=K .
\end{aligned}
$$

Thus, we complete the proof.

Our first theorem of this section shows that the (WCC) is a sufficient and necessary condition for the weak duality to hold.

Theorem 9. (i) The weak duality holds if and only if the family $\left\{F, G, H, \delta_{C}\right\}$ satisfies the $(W C C)_{0}$.

(ii) The stable weak duality holds if and only if the family $\left\{F, G, H, \delta_{C}\right\}$ satisfies the (WCC).

Proof. As assertion (ii) is a global version of assertion (i). Hence, by Proposition 6, it suffices to prove assertion (i). Suppose that the weak duality holds. Let $(0, r) \in K$. Then, by Lemma 8(ii), we have $v(D) \geq-r$ and hence $v(P) \geq-r$, which implies that $(0, r) \in\left(F+G-H+\delta_{C}\right)^{*}$, thanks to Lemma 8(i). Hence, (19) holds; that is, the (WCC) ${ }_{0}$ holds.

Conversely, suppose that the family $\left\{F, G, H, \delta_{C}\right\}$ satisfies the (WCC) $)_{0}$. To show that $v(D) \leq v(P)$, suppose on the contrary that $v(P)<v(D)$. Then, there exists $r \in \mathbb{R}$ such that $v(P)<-r \leq v(D)$. Thus, by the definition of $v(D)$, we have that for each $x^{*} \in \operatorname{dom} H^{*}$, there exist $u^{*} \in \operatorname{dom} F^{*}$ and $v^{*} \in \operatorname{dom} G^{*}$ such that (43) holds. Hence, $(0, r) \in K$ by Lemma 8 (ii), and $(0, r) \in \operatorname{epi}\left(F+G-H+\delta_{C}\right)^{*}$ by the (WCC) $)_{0}$. This together with Lemma 8(i) implies that $v(P) \geq-r$, which contradicts to $v(P)<-r$. Consequently, we have $v(D) \leq v(P)$ and complete the proof.

Theorem 10. (i) The strong duality holds if and only if the family $\left\{F, G, H, \delta_{C}\right\}$ satisfies the $(C C)_{0}$.

(ii) The stable strong duality holds if and only if the family $\left\{F, G, H, \delta_{C}\right\}$ satisfies the $(C C)$.
Proof. As before, it is sufficient to prove assertion (i). Suppose that the strong duality holds. Let $x^{*} \in \operatorname{dom} H^{*}$. Then, $v(P)=$ $v(D)$ and there exist $u^{*} \in \operatorname{dom} F^{*}$ and $v^{*} \in \operatorname{dom} G^{*}$ such that $L\left(x^{*}, u^{*}, v^{*}\right) \geq v(D)$. By Theorem 9(i), (WCC) 0 holds, and so, we only need to verify the following inclusion:

$$
\operatorname{epi}\left(F+G-H+\delta_{C}\right)^{*} \cap(\{0\} \times \mathbb{R}) \subseteq K \cap(\{0\} \times \mathbb{R}) .
$$

To do this, let $(0, r) \in \operatorname{epi}\left(F+G-H+\delta_{C}\right)^{*}$. Then, by Lemma 8(i), we have $v(P) \geq-r$. Hence, $v(D)=v(P) \geq-r$ and $x^{*} \in \operatorname{dom} H^{*}, u^{*} \in \operatorname{dom} F^{*}, v^{*} \in \operatorname{dom} G^{*}$ satisfying (43). This together with Lemma 8 (ii) implies that $(0, r) \in K$ as $x^{*} \in \operatorname{dom} H^{*}$ is arbitrary. Hence, (53) holds and so does the $(\mathrm{CC})_{0}$.

Conversely, suppose that the (CC) $)_{0}$ holds. Then, the family $\left\{f, g, \delta_{C} ; f_{t}, g_{t}: t \in T\right\}$ satisfies (WCC) $)_{0}$, and so $v(D) \leq$ $v(P)$ by Theorem $9(\mathrm{i})$. Thus, to prove the strong duality, it suffices to show that $v(D) \geq v(P)$ and that for each $x^{*} \epsilon$ $\operatorname{dom} H^{*}$ there exist $u^{*} \in \operatorname{dom} F^{*}$ and $v^{*} \in \operatorname{dom} G^{*}$ satisfying $L\left(x^{*}, u^{*}, v^{*}\right) \geq v(D)$. Note that the conclusion holds trivially if $v(P)=-\infty$. Below we only consider the case when $-r:=$ $v(P) \in \mathbb{R}$. By Lemma $8(\mathrm{i}),(0, r) \in \operatorname{epi}\left(F+G-H+\delta_{C}\right)^{*}$, and so $(0, r) \in K$, thanks to the (CC) $)_{0}$. Then, by Lemma 8(ii) and the definition of $v(D)$, we have that $v(D) \geq-r$ and for each $x^{*} \epsilon$ $\operatorname{dom} H^{*}$ there exist $u^{*} \in \operatorname{dom} F^{*}$ and $v^{*} \in \operatorname{dom} G^{*}$ satisfying $L\left(x^{*}, u^{*}, v^{*}\right) \geq-r$. Hence, the strong duality holds. The proof is complete.

Theorem 11. Let $x_{0}$ be a solution of $(P)$. Suppose that the family $\left\{F, G, H, \delta_{C}\right\}$ satisfies the $(M R F)$ at $x_{0}$. Then

$$
\partial H\left(x_{0}\right) \subseteq \partial F\left(x_{0}\right)+\partial G\left(x_{0}\right)+N_{C}\left(x_{0}\right) .
$$

Furthermore, if $\partial H\left(x_{0}\right) \neq \emptyset$, then for each $x^{*} \in \partial H\left(x_{0}\right)$, there exist $u^{*} \in \operatorname{dom} F^{*}$ and $v^{*} \in \operatorname{dom} G^{*}$ such that

$$
v(P)=L\left(x^{*}, u^{*}, v^{*}\right) .
$$

Proof. Since $x_{0}$ is a solution of $(P)$, it follows that

$$
0 \in \partial\left(F+G-H+\delta_{C}\right)\left(x_{0}\right) .
$$

Then, by the (MRF) at $x_{0}$, one has that

$$
0 \in \bigcap_{x^{*} \in \partial H\left(x_{0}\right)}\left(\partial F\left(x_{0}\right)+\partial G\left(x_{0}\right)+N_{C}\left(x_{0}\right)-x^{*}\right),
$$

which is equivalent to (54) holds.

Furthermore, assume that $x^{*} \in \partial H\left(x_{0}\right)$. Then by (54), there exist $u^{*} \in \partial F\left(x_{0}\right), v^{*} \in \partial G\left(x_{0}\right)$, and $w^{*} \in N_{C}\left(x_{0}\right)$ such that

$$
x^{*}=u^{*}+v^{*}+w^{*} .
$$

Since $x^{*} \in \partial H\left(x_{0}\right), u^{*} \in \partial F\left(x_{0}\right), v^{*} \in \partial G\left(x_{0}\right)$, and $w^{*} \in$ $N_{C}\left(x_{0}\right)$, it follows from (7) that

$$
\begin{gathered}
\left\langle x^{*}, x_{0}\right\rangle=H^{*}\left(x^{*}\right)+H\left(x_{0}\right), \\
\left\langle u^{*}, x_{0}\right\rangle=F^{*}\left(u^{*}\right)+F\left(x_{0}\right), \\
\left\langle v^{*}, x_{0}\right\rangle=G^{*}\left(v^{*}\right)+G\left(x_{0}\right), \\
\left\langle w^{*}, x_{0}\right\rangle=\delta_{C}^{*}\left(u^{*}\right) .
\end{gathered}
$$


Hence,

$$
L\left(x^{*}, u^{*}, v^{*}\right)=F\left(x_{0}\right)+G\left(x_{0}\right)-H\left(x_{0}\right)=v(P),
$$

which completes the proof.

Remark 12. In the case when (29) holds, Dinh et al. established the weak duality and strong duality in [5, Theorem 3.2] and the optimality condition in [5, Theorem 3.1] under the assumption that (30) holds. Clearly, by Proposition 7, Theorems 9 and 10 extend and improve [5, Theorem 3.2] and Theorem 11 extends and improves [5, Theorem 3.1].

\section{Optimality Conditions and Dualities for Equilibrium Problem}

Recall the optimization problem (GEP) is defined as in Section 1. Let $x \in C$ and consider the DC optimization problem

$$
\left(\mathscr{P}_{x}\right) \quad p(x):=\inf _{y \in C}\{f(x, y)+\psi(y)\} .
$$

Then, by the definitions of (GEP) and $\left(\mathscr{P}_{x}\right)$, a point $x_{0} \in C$ is a solution of (GEP) if and only if $x_{0}$ is a solution of $\left(\mathscr{P}_{x_{0}}\right)$ (cf. [5, Lemma 3.1]). Moreover, by the definition, we can find that $p(x) \leq \psi(x)$ for each $x \in C$, and, $x_{0}$ is a solution of (GEP) if and only if $p\left(x_{0}\right)=\psi\left(x_{0}\right)$. Hence, the problem of finding solutions of (GEP) can be reduced to the one of finding solutions of the following optimization problem:

$$
(\mathscr{P}) \quad \max _{x \in C} \inf _{y \in C}\left\{f_{x}(y)+\psi(y)-\psi(x)\right\} .
$$

Following [5], we defined the dual problem of $(\mathscr{P})$ by

$$
\begin{gathered}
\max _{x \in C} \inf _{x^{*} \in \operatorname{dom} h^{*}} \max _{u^{*} \in \operatorname{dom} f_{x}^{*}, v^{*} \in \operatorname{dom} g^{*}}\left\{L\left(x, x^{*}, u^{*}, v^{*}\right)\right. \\
-\psi(x)\},
\end{gathered}
$$

where $L: C \times X^{*} \times X^{*} \times X^{*} \rightarrow \overline{\mathbb{R}}$ is defined by

$$
\begin{aligned}
& L\left(x, x^{*}, u^{*}, v^{*}\right) \\
& \quad:=h^{*}\left(x^{*}\right)-f_{x}^{*}\left(u^{*}\right)-g^{*}\left(v^{*}\right)-\delta_{C}^{*}\left(x^{*}-u^{*}-v^{*}\right) .
\end{aligned}
$$

Let $v(\mathscr{P})$ and $v(\mathscr{D})$ denote the optimal values of problems $(\mathscr{P})$ and $(\mathscr{D})$, respectively. Unlike [5], the weak duality (i.e., $v(\mathscr{D}) \leq v(\mathscr{P}))$ does not necessarily holds in general. Recall from Definition 4 that for each $x \in C$, the family $\left\{f_{x}, g, h, \delta_{C}\right\}$ satisfies the $(\mathrm{WCC})_{0}$ if

$$
\begin{aligned}
& \bigcap_{x^{*} \in \operatorname{dom} h^{*}}\left(\text { epi } f_{x}^{*}+\text { epi } g^{*}+\text { epi } \delta_{C}^{*}-\left(x^{*}, h^{*}\left(x^{*}\right)\right)\right) \\
& \cap(\{0\} \times \mathbb{R}) \subseteq \operatorname{epi}\left(f_{x}+g-h+\delta_{C}\right)^{*} \cap(\{0\} \times \mathbb{R})
\end{aligned}
$$

and it satisfies the $(\mathrm{CC})_{0}$ if

$$
\begin{aligned}
& \bigcap_{x^{*} \in \operatorname{dom} h^{*}}\left(\text { epi } f_{x}^{*}+\text { epi } g^{*}+\text { epi } \delta_{C}^{*}-\left(x^{*}, h^{*}\left(x^{*}\right)\right)\right) \\
& \cap(\{0\} \times \mathbb{R})=\operatorname{epi}\left(f_{x}+g-h+\delta_{C}\right)^{*} \cap(\{0\} \times \mathbb{R}) .
\end{aligned}
$$

Then, we have the following theorem.
Theorem 13. (i) Suppose that for each $x \in C$, the family $\left\{f_{x}, g, h, \delta_{C}\right\}$ satisfies the $(W C C)_{0}$. Then, $v(\mathscr{D}) \leq v(\mathscr{P})$.

(ii) Suppose that for each $x \in C$, the family $\left\{f_{x}, g, h, \delta_{C}\right\}$ satisfies the $(C C)_{0}$. Then, $v(\mathscr{D})=v(\mathscr{P})$.

Proof. (i) Since for each $x \in C$, the family $\left\{f_{x}, g, h, \delta_{C}\right\}$ satisfies the (WCC) $)_{0}$, it follows from Theorem 9 (i) that

$$
p(x) \geq \inf _{x^{*} \in \operatorname{dom} h^{*} u^{*} \in \operatorname{dom} f_{x}^{*}, v^{*} \in \operatorname{dom} g^{*}} L\left(x, x^{*}, u^{*}, v^{*}\right) .
$$

Hence, by the definitions of $v(\mathscr{P})$ and $v(\mathscr{D})$, we see that $v(\mathscr{D}) \leq v(\mathscr{P})$.

(ii) Since for each $x \in C$, the family $\left\{f_{x}, g, h, \delta_{C}\right\}$ satisfies the $(\mathrm{CC})_{0}$, it follows from Theorem 10(i) that

$$
p(x)=\inf _{x^{*} \in \operatorname{dom} h^{*}} \max _{u^{*} \in \operatorname{dom} f_{x}^{*}, v^{*} \in \operatorname{dom} g^{*}} L\left(x, x^{*}, u^{*}, v^{*}\right) .
$$

Thus, the result is seen to hold.

The following theorems establish the relationships between the solutions of (GEP) and those of (D). First, we recall that a point $x_{0} \in C$ is said to be a solution of (GEP) if

$$
f\left(x_{0}, y\right)+\psi(y) \geq \psi\left(x_{0}\right) \quad \text { for each } y \in C,
$$

and it is said to be a solution of the dual problem $(\mathscr{D})$ if for each $x^{*} \in \operatorname{dom} h^{*}$, there exist $u^{*}, v^{*} \in X^{*}$ such that

$$
L\left(x_{0}, x^{*}, u^{*}, v^{*}\right)=\psi\left(x_{0}\right)=g\left(x_{0}\right)-h\left(x_{0}\right) .
$$

partially, if for each $x^{*} \in \partial h\left(x_{0}\right)$, there exist $u^{*}, v^{*} \in X^{*}$ such that (70) holds, then $x_{0}$ is said to be a weak solution of problem (D).

Remark 14. (a) Obviously, $x_{0} \in C$ is a solution of (GEP) if and only if $x_{0}$ is a solution of $\left(\mathscr{P}_{x_{0}}\right)$.

(b) Let $x_{0} \in C$. If $h$ is lsc at $x_{0}$, then for each $x^{*} \in \operatorname{dom} h^{*}$, there exist $u^{*} \in \operatorname{dom} f_{x_{0}}^{*}$ and $v^{*} \in \operatorname{dom} g^{*}$ such that

$$
L\left(x_{0}, x^{*}, u^{*}, v^{*}\right) \leq g\left(x_{0}\right)-h\left(x_{0}\right) .
$$

Consequently, $x_{0}$ is a solution of the dual problem (D) if and only if for each $x^{*} \in \operatorname{dom} h^{*}$, there exist $u^{*}, v^{*} \in X^{*}$ such that

$$
L\left(x_{0}, x^{*}, u^{*}, v^{*}\right) \geq g\left(x_{0}\right)-h\left(x_{0}\right) .
$$

In fact, let $x^{*} \in \operatorname{dom} h^{*}, u^{*} \in \operatorname{dom} f_{x_{0}}^{*}$, and $v^{*} \in \operatorname{dom} g^{*}$. Then by the Young-Fenchel inequality (6), it is easy to see that

$$
\begin{aligned}
L & \left(x_{0}, x^{*}, u^{*}, v^{*}\right) \\
& \leq h^{*}\left(x^{*}\right)-\left\langle x^{*}, x_{0}\right\rangle+g\left(x_{0}\right)+f_{x_{0}}\left(x_{0}\right) \\
& \leq \inf _{x^{*} \in \operatorname{dom} h^{*}}\left\{h^{*}\left(x^{*}\right)-\left\langle x^{*}, x_{0}\right\rangle\right\}+g\left(x_{0}\right)+f_{x_{0}}\left(x_{0}\right) \\
& =-(\operatorname{cl} h)\left(x_{0}\right)+g\left(x_{0}\right) \\
& =g\left(x_{0}\right)-h\left(x_{0}\right) .
\end{aligned}
$$

Hence, (71) holds. However, (71) does not necessarily hold in general as will be showen in the following example. 
Example 15. Let $X:=\mathbb{R}$ and $C:=(-\infty, 0]$. Let $f: \mathbb{R} \times \mathbb{R} \rightarrow$ $\overline{\mathbb{R}}$ and $g, h: \mathbb{R} \rightarrow \overline{\mathbb{R}}$ be defined by $f:=0, g:=\delta_{[0,+\infty)}$, and

$$
h(x):= \begin{cases}0 & x<0 \\ 1 & x=0 \\ +\infty & x>0\end{cases}
$$

Let $x_{0}=0$. Then, $g\left(x_{0}\right)-h\left(x_{0}\right)=-1$, and $h$ is not lsc at $x_{0}$. Clearly, $f_{x_{0}}^{*}=\delta_{\{0\}}, g^{*}=\delta_{(-\infty, 0]}$, and $h^{*}=\delta_{C}^{*}=\delta_{[0,+\infty)}$. Then, $\operatorname{dom} f_{x_{0}}^{*}=\{0\}$, dom $g^{*}=(-\infty, 0]$, and $\operatorname{dom} h^{*}=\operatorname{dom} \delta_{C}^{*}=$ $[0,+\infty)$. Let $x^{*}=0$. Then, for each $u^{*} \in \operatorname{dom} f_{x_{0}}^{*}$ and $v^{*} \in$ dom $g^{*}$, one has that

$$
L\left(x_{0}, x^{*}, u^{*}, v^{*}\right)=-g^{*}\left(v^{*}\right)-\delta_{C}^{*}\left(-v^{*}\right)=0 ;
$$

this implies that (71) does not hold.

By Theorem 11, we obtain the the following theorem straightforwardly, which was established in [5, Theorem 4.2] under the assumptions that (29) holds and

$$
\text { epi } f_{x}^{*}+\text { epi } g^{*}+\text { epi } \delta_{C}^{*} \text { is weak }{ }^{*} \text {-closed. }
$$

Thus, by Proposition 7, our Theorem 16 improves the corresponding result in [5, Theorem 4.2].

Theorem 16. Let $x_{0} \in C$ such that $\partial H\left(x_{0}\right) \neq \emptyset$. Suppose that the family $\left\{f_{x_{0}}, g, h, \delta_{C}\right\}$ satisfies the $(M R F)$ at $x_{0}$. If $x_{0}$ is a solution of $(G E P)$, then $x_{0}$ is a weak solution of $(\mathscr{D})$.

Theorem 17. Let $x_{0} \in C$. Suppose that $h$ is lsc and that the family $\left\{f_{x_{0}}, g, h, \delta_{C}\right\}$ satisfies the $(C C)_{0}$. Then, $x_{0}$ is a solution of $(G E P)$ if and only if $x_{0}$ is a solution of the dual problem (D).

Proof. Suppose that $x_{0}$ is a solution of (GEP). Then, $x_{0}$ is a solution of $\left(\mathscr{P}_{x_{0}}\right)$. Hence,

$$
v\left(\mathscr{P}_{x_{0}}\right)=f_{x_{0}}\left(x_{0}\right)+g\left(x_{0}\right)-h\left(x_{0}\right)=g\left(x_{0}\right)-h\left(x_{0}\right) .
$$

Since the family $\left\{f_{x_{0}}, g, h, \delta_{C}\right\}$ satisfies the $(\mathrm{CC})_{0}$, it follows from Theorem 10 that

$$
v\left(\mathscr{P}_{x_{0}}\right)=\inf _{x^{*} \in \operatorname{dom} h^{*}} \max _{u^{*} \in \operatorname{dom} f_{x_{0}}^{*}, v^{*} \in \operatorname{dom} g^{*}} L\left(x_{0}, x^{*}, u^{*}, v^{*}\right),
$$

which implies that for each $x^{*} \in \operatorname{dom} h^{*}$, there exist $u^{*} \in$ $\operatorname{dom} f_{x_{0}}^{*}$ and $v^{*} \in \operatorname{dom} g^{*}$ such that

$$
L\left(x_{0}, x^{*}, u^{*}, v^{*}\right) \geq v\left(P_{x_{0}}\right)
$$

Moreover, since $h$ is lsc at $x_{0}$, it follows from Remark 14(b) that

$$
L\left(x_{0}, x^{*}, u^{*}, v^{*}\right) \leq g\left(x_{0}\right)-h\left(x_{0}\right)=v\left(P_{x_{0}}\right) .
$$

Hence, (70) holds. This means that $x_{0}$ is a solution of the dual problem (D).
Conversely, suppose that $x_{0}$ is a solution of the dual problem (D). Let $x^{*} \in \operatorname{dom} h^{*}$. Then, there exist $u^{*} \in \operatorname{dom} f_{x_{0}}^{*}$ and $v^{*} \in \operatorname{dom} g^{*}$ such that (70) holds. Note by the YoungFenchel inequality (6) that for each $x \in C$,

$$
L\left(x_{0}, x^{*}, u^{*}, v^{*}\right) \leq h^{*}\left(x^{*}\right)-\left\langle x^{*}, x\right\rangle+g(x)+f_{x_{0}}(x) .
$$

Then by (70), one has that for each $x \in C$,

$$
g\left(x_{0}\right)-h\left(x_{0}\right) \leq h^{*}\left(x^{*}\right)-\left\langle x^{*}, x\right\rangle+g(x)+f_{x_{0}}(x) .
$$

Since the above inequality holds for each $x^{*} \in \operatorname{dom} h^{*}$, it follows that

$$
\begin{aligned}
& g\left(x_{0}\right)-h\left(x_{0}\right) \\
& \leq \inf _{x^{*} \in \operatorname{dom} h^{*}}\left\{h^{*}\left(x^{*}\right)-\left\langle x^{*}, y\right\rangle\right\} \\
& \quad+g(x)+f_{x_{0}}(x) \\
& =-(\operatorname{cl} h)(x)+g(x)+f_{x_{0}}(x) .
\end{aligned}
$$

Consequently, by the lower semicontinuity of $h$, one has that for each $x \in C$,

$$
g\left(x_{0}\right)-h\left(x_{0}\right) \leq f_{x_{0}}(x)+g(x)-h(x),
$$

and hence $g\left(x_{0}\right)-h\left(x_{0}\right) \leq p\left(x_{0}\right)$. This implies that $g\left(x_{0}\right)-$ $h\left(x_{0}\right)=p\left(x_{0}\right)$ since $g\left(x_{0}\right)-h\left(x_{0}\right) \geq p\left(x_{0}\right)$ holds automatically. Thus, $x_{0}$ is a solution of (GEP).

Remark 18. Theorem 17 was established in [5, Theorems 4.3 and 4.4] under the assumptions that (29) and (76) hold. Thus, our Theorem 16 extends the corresponding result in [5, Theorems 4.3 and 4.4] to suit the case when $g, h$ are not lsc and $C$ is not closed.

Below we will give a upper estimate for the Fréchet subdifferential of the function $p$ defined in (61). We first recall from [18] or [19, page 90] that the Fréchet subdifferential of $\phi$ at a point $x_{0} \in \operatorname{dom} \phi$ with $\left|\phi\left(x_{0}\right)\right|<\infty$ is defined by

$$
\begin{aligned}
& \widehat{\partial} \phi\left(x_{0}\right) \\
& :=\left\{x^{*} \in X^{*}: \liminf _{x \rightarrow x_{0}} \frac{\phi(x)-\phi\left(x_{0}\right)-\left\langle x^{*}, x-x_{0}\right\rangle}{\left\|x-x_{0}\right\|} \geq 0\right\},
\end{aligned}
$$

where $\phi: X \rightarrow \mathbb{R} \cup\{ \pm \infty\}$ is an extended real-valued function.

Theorem 19. Let $x_{0} \in C$ be a solution of $(G E P)$. Suppose that the family $\left\{g, \delta_{C}\right\}$ satisfies the $(M R F)$ at $x_{0}$; that is,

$$
\partial\left(g+\delta_{C}\right)\left(x_{0}\right)=\partial g\left(x_{0}\right)+N_{C}\left(x_{0}\right) .
$$

Then for each $\gamma>0$, one has that

$$
\widehat{p}\left(x_{0}\right) \subseteq \bigcap_{x^{*} \in \partial h\left(x_{0}\right)}\left(\partial g\left(x_{0}\right)+N_{C}\left(x_{0}\right)-x^{*}\right)+\gamma \mathbb{B}^{*},
$$

where $\mathbb{B}^{*}$ denotes the unit ball in $X^{*}$. 
Proof. Let $x_{0} \in C$. If $\partial H\left(x_{0}\right)=\emptyset$, then (87) holds trivially. Below let $u^{*} \in \widehat{\partial} p\left(x_{0}\right)$ and $x^{*} \in \partial h\left(x_{0}\right)$. Let $\gamma>0$. Then, by the definition of of Fréchet subdifferential of $p$ at $x_{0}$, there exists $\eta>0$ such that

$$
\begin{aligned}
& p(x)-p\left(x_{0}\right)-\left\langle u^{*}, x-x_{0}\right\rangle+\gamma\left\|x-x_{0}\right\| \\
& \quad \geq 0 \text { for each } x \in x_{0}+\eta \mathbb{B},
\end{aligned}
$$

where $\mathbb{B}$ denotes the unit ball in $X$. Since $x_{0}$ is a solution of (GEP), it follows that $p\left(x_{0}\right)=g\left(x_{0}\right)-h\left(x_{0}\right)$. Note that $p(x) \leq$ $g(x)-h(x)$ holds for each $x \in C$. Then

$$
\begin{aligned}
0 \leq & g(x)-h(x)-g\left(x_{0}\right)+h\left(x_{0}\right) \\
& -\left\langle u^{*}, x-x_{0}\right\rangle+\gamma\left\|x-x_{0}\right\| \\
\leq & g(x)-g\left(x_{0}\right)-\left\langle u^{*}+x^{*}, x-x_{0}\right\rangle \\
& +\gamma\left\|x-x_{0}\right\|,
\end{aligned}
$$

where the last inequality holds because $x^{*} \in \partial h\left(x_{0}\right)$. Define $\phi: X \rightarrow \overline{\mathbb{R}}$ by

$$
\phi(x):=g(x)-g\left(x_{0}\right)-\left\langle u^{*}+x^{*}, x-x_{0}\right\rangle+\gamma\left\|x-x_{0}\right\| .
$$

Then, $\phi$ is a proper convex function on $X$, and $x_{0}$ is a solution to the following convex programming:

$$
\begin{aligned}
& \text { Min } \phi(x), \\
& \text { s.t. } x \in C \cap\left(x_{0}+\eta \mathbb{B}\right) .
\end{aligned}
$$

Hence, $0 \in \partial\left(\phi+\delta_{C \cap\left(x_{0}+\eta \mathbb{B}\right)}\right)\left(x_{0}\right)$, and

$$
0 \in \partial\left(g+\delta_{C \cap\left(x_{0}+\eta \mathbb{B}\right)}\right)\left(x_{0}\right)-\left(u^{*}+x^{*}\right)+\gamma \mathbb{B}^{*} .
$$

Note that $x_{0}$ is an interior point of the set $x_{0}+\eta \mathbb{B}$; then the function $\delta_{x_{0}+\eta \mathbb{B}}$ is continuous at $x_{0}$. Hence,

$$
\partial\left(g+\delta_{\mathrm{C} \cap\left(x_{0}+\eta \mathbb{B}\right)}\right)\left(x_{0}\right)=\partial\left(g+\delta_{C}\right)\left(x_{0}\right) .
$$

This together with (86) and (92) implies that

$$
0 \in \partial g\left(x_{0}\right)+N_{C}\left(x_{0}\right)-\left(u^{*}+x^{*}\right)+\gamma \mathbb{B}^{*} ;
$$

that is,

$$
u^{*} \in \partial g\left(x_{0}\right)+N_{C}\left(x_{0}\right)-x^{*}+\gamma \mathbb{B}^{*},
$$

Which implies that (87) holds by the arbitrariness of $x^{*}$. The proof is complete.

\section{Conflict of Interests}

The authors declare that there is no conflict of interests regarding the publication of this paper.

\section{Acknowledgments}

D. H. Fang was supported in part by the National Natural Science Foundation of China (Grant no. 11101186) and supported in part by the Scientific Research Fund of Hunan Provincial Education Department (Grant no. 13B095).

\section{References}

[1] C. Zălinescu, Convex Analysis in General Vector Spaces, World Scientific Publishing, River Edge, NJ, USA, 2002.

[2] M. A. Noor and K. I. Noor, "On equilibrium problems," Applied Mathematics E-Notes, vol. 4, pp. 125-132, 2004.

[3] G. Bigi, M. Castellani, and G. Kassay, "A dual view of equilibrium problems," Journal of Mathematical Analysis and Applications, vol. 342, no. 1, pp. 17-26, 2008.

[4] E. Blum and W. Oettli, "From optimization and variational inequalities to equilibrium problems," The Mathematics Student, vol. 63, no. 1-4, pp. 123-145, 1994.

[5] N. Dinh, J. J. Strodiot, and V. H. Nguyen, "Duality and optimality conditions for generalized equilibrium problems involving DC functions," Journal of Global Optimization, vol. 48, no. 2, pp. 183-208, 2010.

[6] D. H. Fang, C. Li, and K. F. Ng, "Constraint qualifications for extended Farkas's lemmas and Lagrangian dualities in convex infinite programming," SIAM Journal on Optimization, vol. 20, no. 3, pp. 1311-1332, 2009.

[7] A. N. Iusem and W. Sosa, "Iterative algorithms for equilibrium problems," Optimization, vol. 52, no. 3, pp. 301-316, 2003.

[8] L. D. Muu, V. H. Nguyen, and N. V. Quy, "On Nash-Cournot oligopolistic market equilibrium models with concave cost functions," Journal of Global Optimization, vol. 41, no. 3, pp. 351364, 2008.

[9] L. D. Muu and W. Oettli, "Convergence of an adaptive penalty scheme for finding constrained equilibria," Nonlinear Analysis. Theory, Methods \& Applications, vol. 18, no. 12, pp. 1159-1166, 1992.

[10] T. T. V. Nguyen, J. J. Strodiot, and V. H. Nguyen, "A bundle method for solving equilibrium problems," Mathematical Programming, vol. 116, no. 1-2, pp. 529-552, 2009.

[11] I. V. Konnov and S. Schaible, "Duality for equilibrium problems under generalized monotonicity," Journal of Optimization Theory and Applications, vol. 104, no. 2, pp. 395-408, 2000.

[12] J. E. Martínez-Legaz and W. Sosa, "Duality for equilibrium problems," Journal of Global Optimization, vol. 35, no. 2, pp. 311319, 2006.

[13] F. M. O. Jacinto and S. Scheimberg, "Duality for generalized equilibrium problem," Optimization, vol. 57, no. 6, pp. 795-805, 2008.

[14] L. Altangerel, R. I. Boţ, and G. Wanka, "On the construction of gap functions for variational inequalities via conjugate duality," Asia-Pacific Journal of Operational Research, vol. 24, no. 3, pp. 353-371, 2007.

[15] L. Altangerel, R. I. Boţ, and G. Wanka, "On gap functions for equilibrium problems via Fenchel duality," Pacific Journal of Optimization, vol. 2, no. 3, pp. 667-678, 2006.

[16] U. Mosco, "Dual variational inequalities," Journal of Mathematical Analysis and Applications, vol. 40, pp. 202-206, 1972.

[17] D. H. Fang, C. Li, and X. Q. Yang, "Stable and total Fenchel duality for DC optimization problems in locally convex spaces," SIAM Journal on Optimization, vol. 21, no. 3, pp. 730-760, 2011.

[18] B. S. Mordukhovich, N. M. Nam, and N. D. Yen, "Fréchet subdifferential calculus and optimality conditions in nondifferentiable programming," Optimization, vol. 55, no. 5-6, pp. 685-708, 2006.

[19] B. S. Mordukhovich, Variational Analysis and Generalized Differentiation, vol. 330, Springer, Berlin, Germany, 2006. 


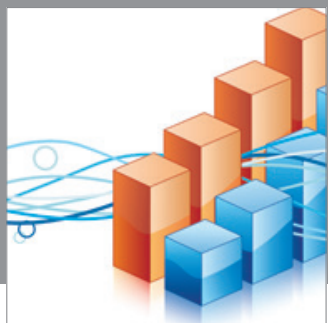

Advances in

Operations Research

mansans

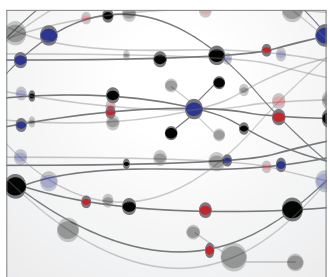

The Scientific World Journal
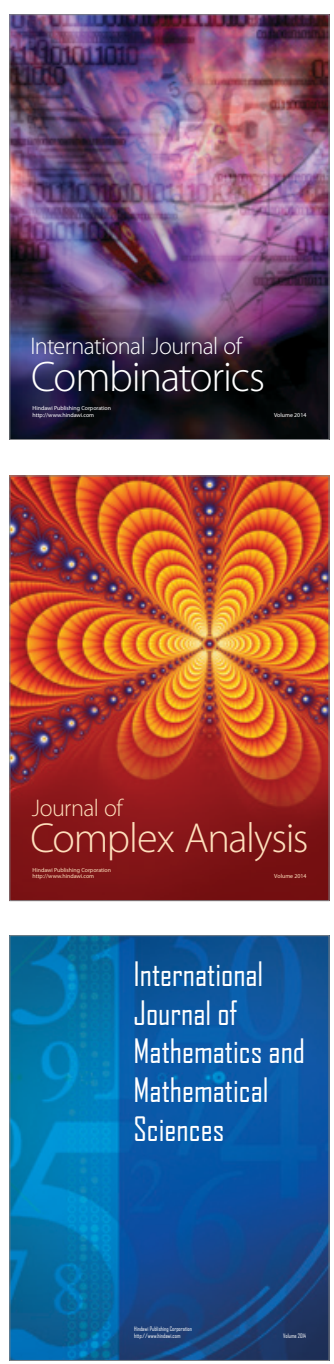
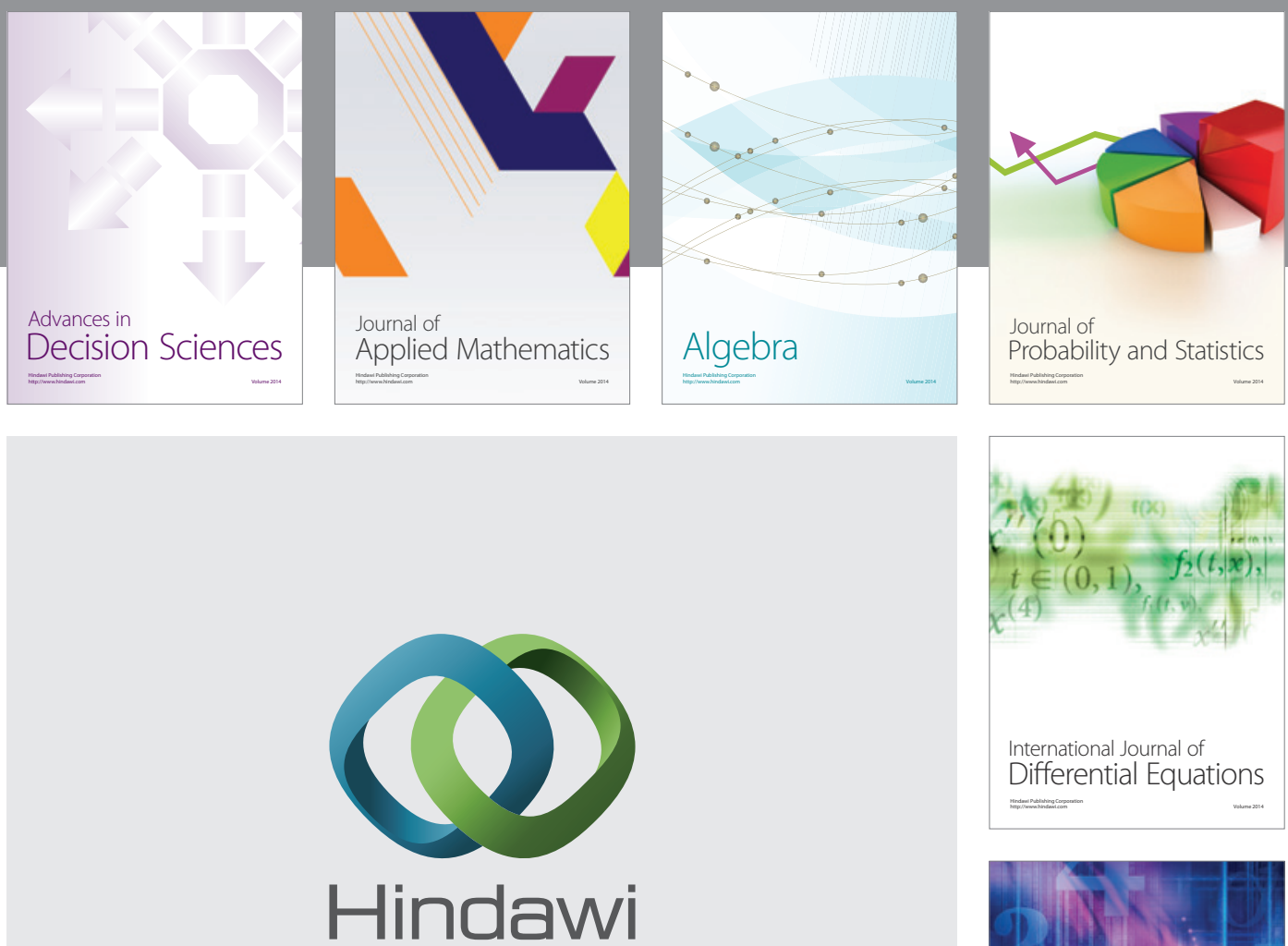

Submit your manuscripts at http://www.hindawi.com
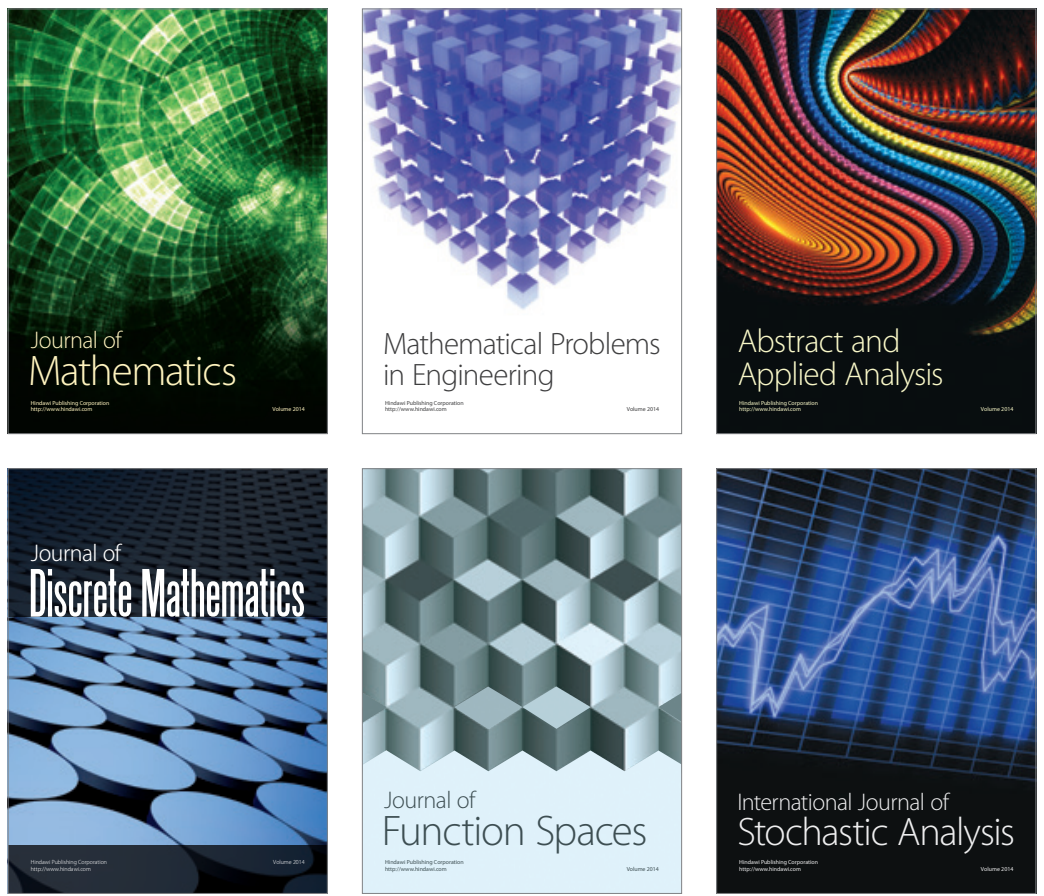

Journal of

Function Spaces

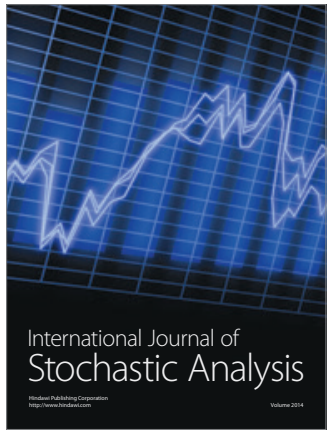

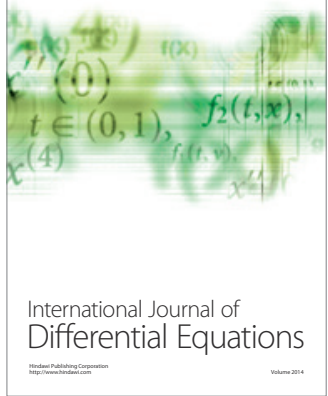
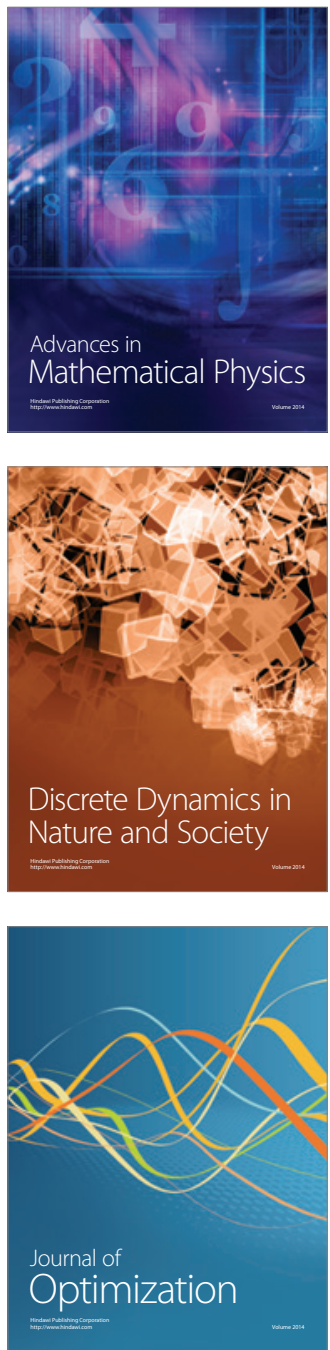